\title{
Ultrasound-Guided Tendon Fenestration
}

\author{
Mary M. Chiavaras, MD, PhD ${ }^{1}$ Jon A. Jacobson, $\mathrm{MD}^{2}$ \\ ${ }^{1}$ Department of Radiology, McMaster University, Hamilton General \\ Hospital, Hamilton, Ontario, Canada \\ 2 Department of Radiology, University of Michigan, Ann Arbor, \\ Michigan
}

Address for correspondence Mary M. Chiavaras, MD, PhD, Department of Radiology, McMaster University, Hamilton General Hospital, Diagnostic Imaging, 237 Barton St. E, Hamilton, ON, Canada L8L 2X2 (e-mail: chiavaras@hhsc.ca).

Semin Musculoskelet Radiol 2013;17:85-90.

\begin{abstract}
Keywords

- elbow

- ultrasound

- tendinosis

- epicondylitis
\end{abstract}

Pain related to chronic tendon abnormalities can be debilitating and interfere with personal, recreational, and occupational activities. Commonly involved sites include the rotator cuff, ${ }^{1,2}$ the common extensor tendon of the elbow (lateral epicondylitis), ${ }^{3}$ the patellar tendon (jumper's knee), ${ }^{4}$ the gluteal tendons (greater trochanteric pain syndrome), ${ }^{5}$ and the Achilles tendon, ${ }^{6}$ among others. Conservative noninvasive treatments have typically included rest, nonsteroidal anti-inflammatory medication, and physical therapy; however, these treatments are often unsuccessful or short lived.

Several minimally invasive interventional procedures have also been used to treat chronic tendon problems that include tendon fenestration (also termed dry needling or tenotomy) and injections of various substances including corticosteroids, hyperosmolar dextrose, autologous whole blood, and autologous platelet-rich plasma (PRP). These procedures are generally performed using ultrasound guidance, which increases accuracy and decreases potential complications. In some cases, surgical tenotomy may be considered as another treatment for tendinosis.

Despite the number of potential treatments, there is no clear evidence that one treatment is superior to the others.
The key to deciding on the appropriate treatment is to clearly understand the underlying tendon pathology.

\section{Tendinosis}

Although historically termed tendinitis, it is now well known that chronic tendon symptoms are not truly inflammatory but rather are related to tendon degeneration with a possible superimposed tendon tear. ${ }^{1-6}$ Although active inflammation occurs after an acute injury, this inflammatory response decreases by 10 days postinjury as the proliferative phase of healing begins. ${ }^{7}$ Chronic tendon symptoms are associated with tendon degeneration, which includes mucoid degeneration and chondroid metaplasia, with possible interstitial tears. Such changes have been documented in the supraspinatus, ${ }^{2}$ common extensor tendon of the elbow, ${ }^{3}$ proximal patellar tendon, ${ }^{4}$ and Achilles tendon. ${ }^{6}$ The term tendinosis (or tendinopathy) is used in this situation to indicate that inflammation is not a predominant feature.

Characteristic imaging features of tendinosis are identified on both MR imaging and ultrasound. At MR imaging, the normal low signal intensity tendon appears as intermediate
Issue Theme Musculoskeletal Ultrasound Update; Guest Editor, Jon A. Jacobson, MD.
Copyright (c) 2013 by Thieme Medical Publishers, Inc., 333 Seventh Avenue, New York, NY 10001, USA. Tel: +1(212) 584-4662. 

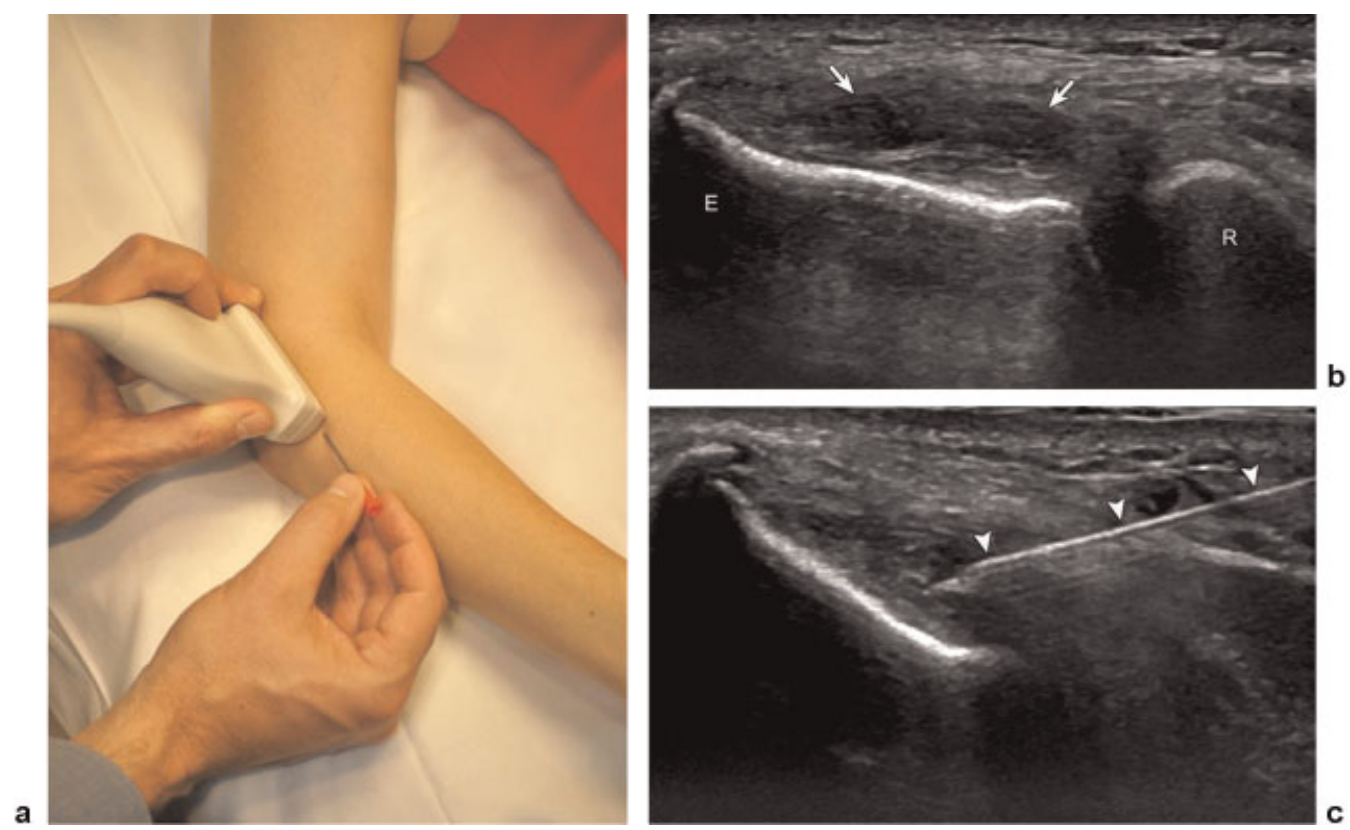

Fig. 1 (a) Photograph shows needle and transducer position for a simulated common extensor tendon fenestration. The needle is positioned in plane, along the long axis of the tendon, parallel to the transducer, from distal to proximal. (b, c) A 47-year-old man with tendinosis and interstitial tear of common extensor tendon and subsequent fenestration. (b) Ultrasound image over lateral elbow long axis to common extensor tendon (right side of image is distal) shows abnormal hypoechoic swelling of the proximal tendon with interstitial anechoic clefts (arrows). E, lateral epicondyle; R, radial head. (c) Ultrasound image long axis to common extensor tendon shows 22-gauge needle (arrowheads) used for fenestration with distal tip area of tendinosis and interstitial tear (also see video 1d).

signal intensity approximately equal to muscle with tendon swelling. ${ }^{2}$ On ultrasound, the normally hyperechoic and fibrillar tendon appears hypoechoic and thickened with loss of the normal fibrillar pattern (-Figs. 1-4). ${ }^{8}$ Superimposed interstitial tears appear as more focal fluid signal clefts on MR imaging and focal anechoic areas of tendon fiber disruption on ultrasound. ${ }^{8,9}$ Additional features may include adjacent cortical irregularity at the tendon enthesis and intratendinous calcifications, optimally demonstrated with ultrasound. $^{8}$

With some tendons, such as the common extensor tendon of the elbow, the patellar tendon, and the Achilles tendon, increased flow may be demonstrated on color and power Doppler imaging ( $\mathbf{- F i g . ~ 4 b )}$ ). Such hyperemia has been shown to represent neovascularity and not inflammation. ${ }^{3,4,6}$ Blood flow is generally not present within a normal tendon on color or power Doppler imaging. ${ }^{10}$ With the Achilles tendon, hyperemia within an area of tendinosis has been associated with pain. ${ }^{11}$ Although increased vascularity tends to be associated with increased severity and pain, a clear association with chronicity, severity, or symptoms may not present in all cases. ${ }^{12}$

\section{Ultrasound-Guided Tendon Injections}

A variety of ultrasound-guided nonsurgical minimally invasive interventional procedures have been used to treat tendinosis. Although a detailed discussion about each of these techniques is beyond the scope and focus of this article, an overview of such techniques is relevant and discussed briefly.

\section{Corticosteroids}

One of the most common treatments has traditionally been corticosteroid injection over the surface of abnormal tendon; however, studies have demonstrated that, although corticosteroid injections can be effective, they are often short lived. For example, peritendinous corticosteroid injection of the common extensor tendon of the elbow has been shown to provide short-term symptom relief ( $<8$ weeks), with less benefit at 26 weeks and beyond, and even negative outcomes at 6 months and 1 year. ${ }^{13}$ With the rotator cuff, there is conflicting evidence of any real short-term improvement with corticosteroid injection. ${ }^{13}$ Ultrasound-guided peritendinous corticosteroid injection of hamstring tendinosis resulted in $50 \%$ of patients with improved symptoms at 1 month and only $24 \%$ of patients $>6$ months after injection with no reported complications. ${ }^{14} \mathrm{~A}$ similar response has been described with ultrasound-guided peritendinous corticosteroid injection of the gluteal tendons about the greater trochanter, with only $55 \%$ of patients having pain reduction after treatment. ${ }^{15}$

The disadvantage of corticosteroid injection superficial to a tendon is that the underlying tendon abnormality is not directly treated, which may explain the relatively short-lived improvement of patient symptoms. Temporary symptom relief after corticosteroid injection is not completely understood, although altered release of toxins as well as inhibition of collagen, extracellular matrix molecules, and granulation tissue have been proposed. ${ }^{13}$ Moreover, the use of corticosteroids in the setting of tendinosis as an anti-inflammatory medication is questionable because inflammation is not considered to be part of the underlying pathophysiologic process. 

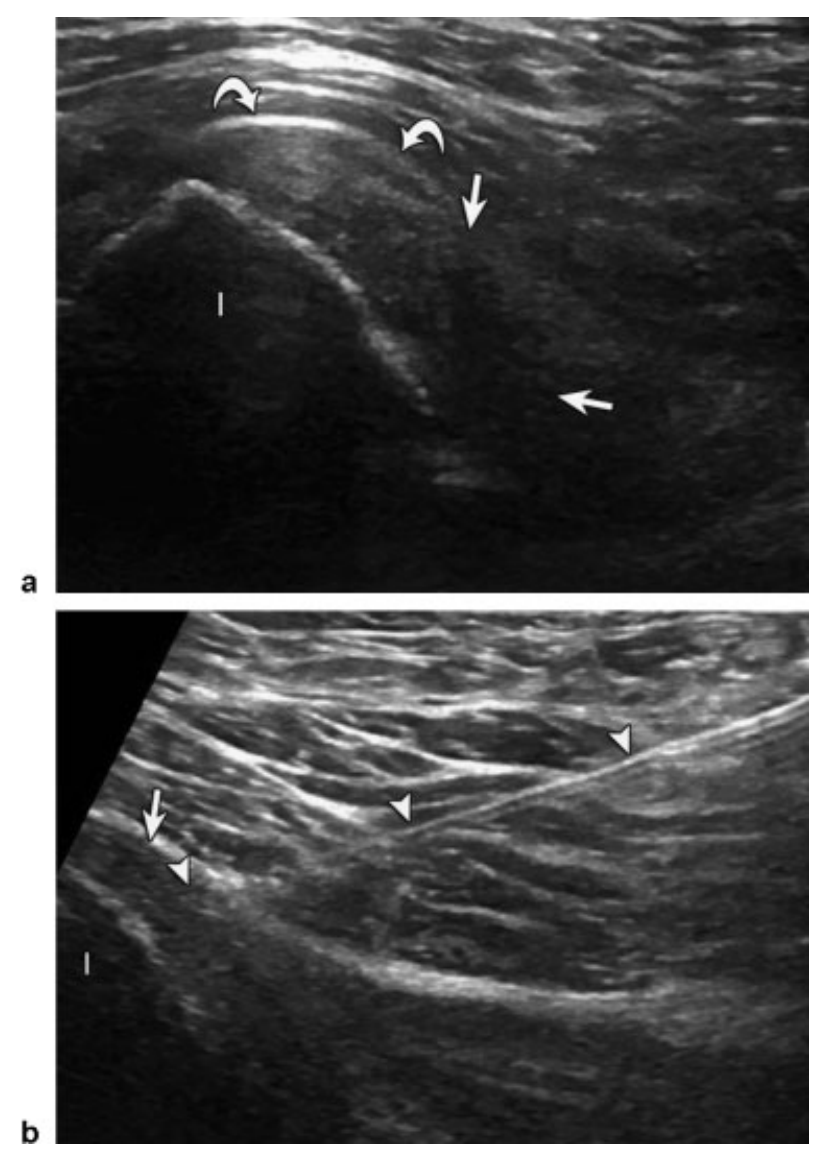

Fig. 2 A 46-year-old man with tendinosis of the proximal semimembranosus and conjoint tendon and subsequent fenestration. (a) UItrasound image over posterior gluteal region short axis to proximal hamstring tendons shows abnormal hypoechoic swelling of the semimembranosus tendon (arrows) and to a lesser extent the conjoint tendon (curved arrows) (I, ischial tuberosity; right side of image is lateral). (b) Ultrasound image long axis to semimembranosus tendon (right side of image is distal) shows 20-gauge needle (arrowheads) used for fenestration with distal tip in area of tendinosis (arrow) (also see video $2 \mathrm{c}$ ).

It should be emphasized that injection of corticosteroids directly into a tendon has been shown to weaken a tendon and predispose to rupture due to inhibition of tenocyte proliferation, reduction in collagen fascicle strength, and reduction of inflammation that assists with the healing process. ${ }^{16}$ Other potential complications of corticosteroids are fat necrosis and depigmentation if injected into the subcutaneous tissues. ${ }^{16}$ These complications underscore the importance of ultrasound guidance to ensure accurate placement of the corticosteroid. Lastly, the potential systemic effects of corticosteroids, such as suppression of adrenocorticotropic hormone and increased blood glucose levels in patients with diabetes, must also be considered. ${ }^{16}$

\section{Prolotherapy}

In contrast to the injection of corticosteroids adjacent to an abnormal tendon, several ultrasound-guided interventional techniques directly target the tendon abnormality. One such treatment is prolotherapy, which involves injection of an
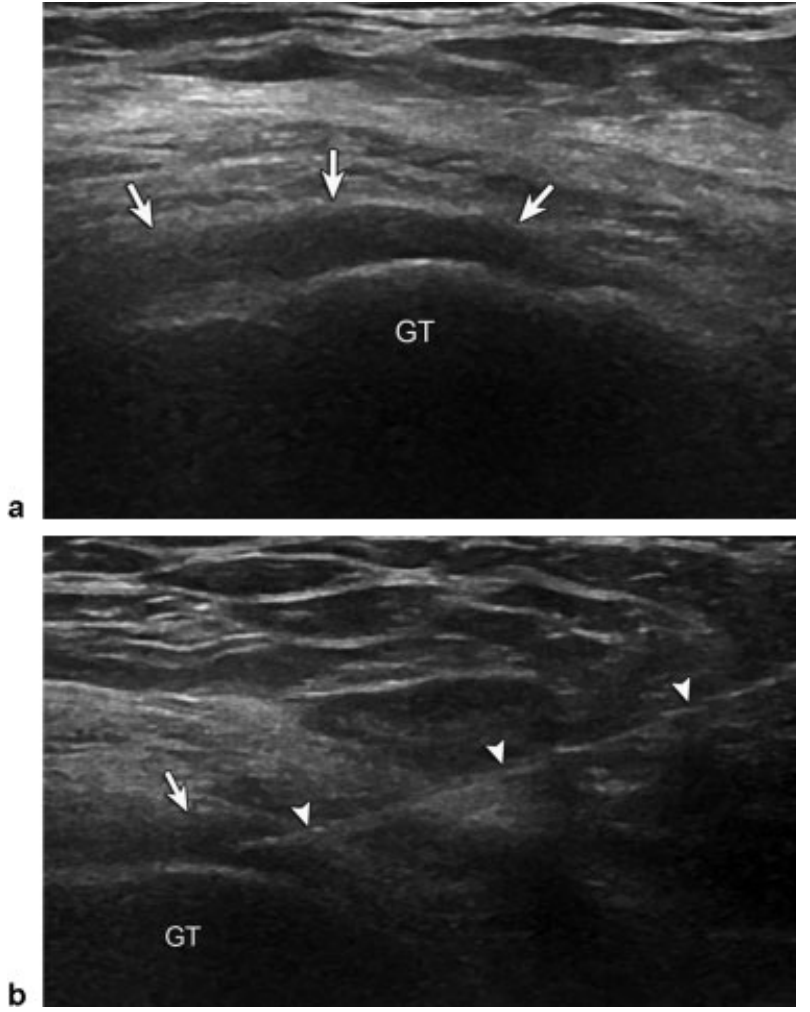

Fig. 3 A 74-year-old man with tendinosis of the gluteus medius and subsequent fenestration. (a) Ultrasound image over lateral hip long axis to gluteus medius tendon (right side of image is distal) shows abnormal hypoechoic swelling of the gluteus medius tendon (arrows) over the lateral facet of the greater trochanter (GT). (b) Ultrasound image long axis to gluteus medius tendon shows 20-gauge needle (arrowheads) used for fenestration with distal tip in area of tendinosis (arrow) (also see video 3c).

irritant, most commonly hyperosmolar dextrose, into the area of tendinosis. ${ }^{17,18}$ The rationale behind this treatment is that the irritant improves symptoms by either (1) causing inflammation, which introduces growth factors that promote healing, or (2) by acting as a vascular sclerosing agent. ${ }^{17}$

\section{Autologous Blood Injection}

In autologous whole-blood tendon injection, peripheral blood is drawn from the patient's arm and reinjected into the pathologic tendon using ultrasound guidance. ${ }^{19}$ The rationale is that autologous platelets within the whole blood, placed at the site of tendon abnormality, will increase the concentration of growth factors to the region and promote healing (see later article by Kenneth Lee in this issue).

With the use of a centrifuge, the platelet component of the patient's blood (PRP) can be isolated, concentrated, and then reinjected into an area of tendinosis as another form of ultrasound-guided treatment. ${ }^{19}$ The rationale for the use of PRP over whole blood is the more concentrated the platelets, the better the clinical response.

The potential benefits of autologous whole blood versus PRP injection for the treatment of tendinosis are currently being debated. ${ }^{20,21}$ Of note, both types of injections are often combined with tendon fenestration. ${ }^{22,23}$ It is interesting that 

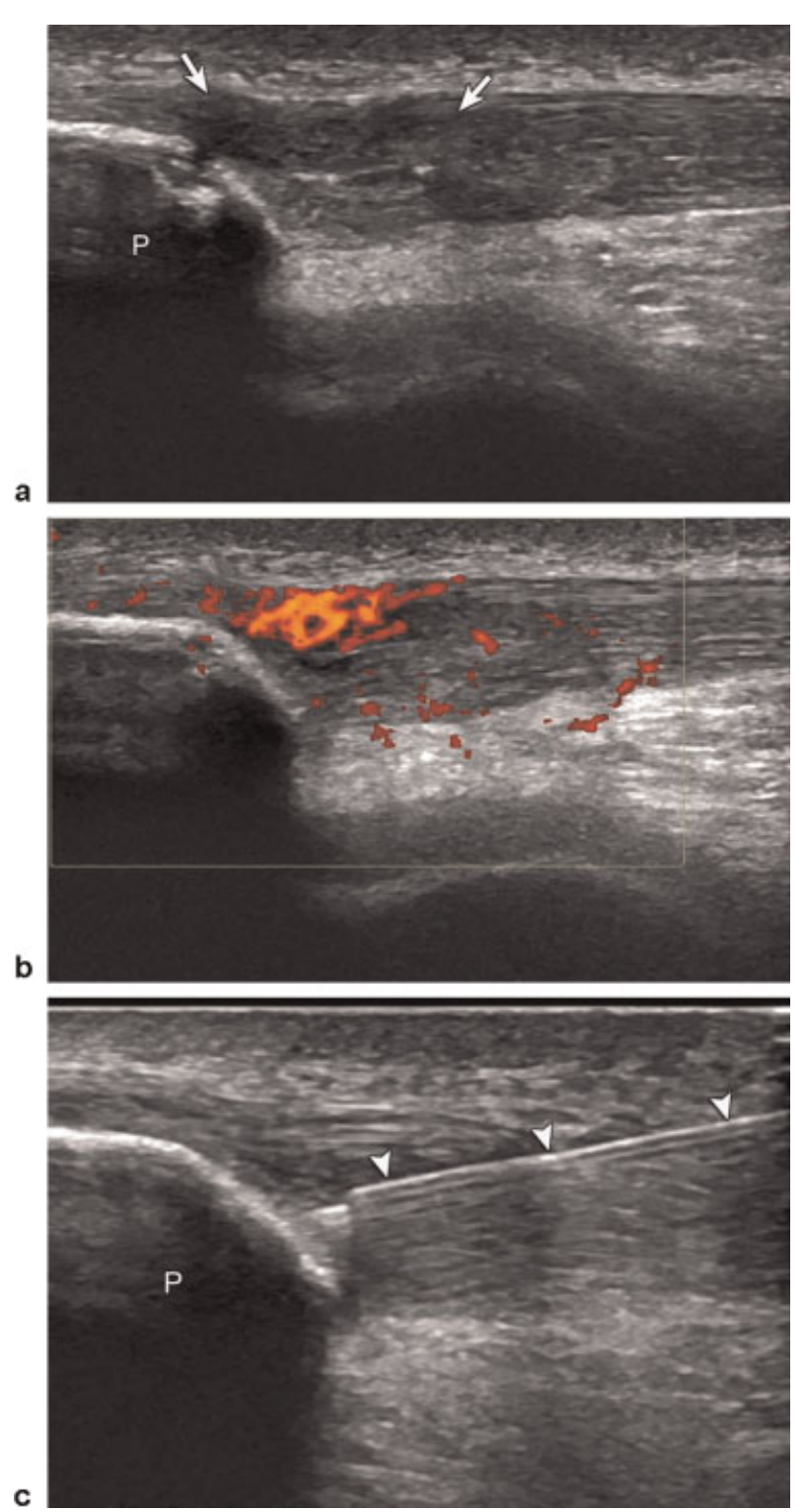

Fig. 4 A 22-year-old man with tendinosis of patellar tendon and subsequent fenestration. (a) Ultrasound image over anterior knee long axis to patellar tendon (right side of image is distal) shows abnormal hypoechoic swelling of patellar tendon (arrows) with calcification and cortical irregularity of the patella (P). (b) Corresponding power Doppler image shows increased blood flow. (c) Ultrasound image long axis to patellar tendon shows 22-gauge needle (arrowheads) used for fenestration with distal tip in area of tendinosis (also see video $4 d$ ).

each of these potential ultrasound-guided tendon treatments involves inserting a needle into the tendon, and therefore one opinion is that simply placing the needle into the tendon may be the primary reason that the tendon improves. This is the basis of ultrasound-guided tendon fenestration.

\section{Tendon Fenestration}

The use of a needle as a treatment for tendinosis is termed tendon fenestration. This procedure has also been called dry needling to emphasize that the procedure does not involve the injection of autologous whole blood or PRP, although often autologous blood injections are combined with tendon fenestration. $^{22,23}$ Tendon fenestration has also been referred to as tenotomy, although some believe this term is less appropriate because the term tenotomy may imply a tendon release or lengthening as described in the orthopedic surgery literature. $^{24,25}$

The use of a needle to treat tendinosis has been used for decades. Although initially performed blindly using palpation, the use of ultrasound guidance is preferred to ensure accurate placement of the needle into the abnormal portion of the tendon, avoid other structures, and reduce complications. The theory behind this procedure is that repetitively passing the needle though the area of tendinosis disrupts the chronic degenerative process, causes bleeding and inflammation, and locally increases growth factors and other substances that promote healing.

One of the most common tendons treated with fenestration is the common extensor tendon of the elbow, where it has been shown that $80 \%$ of patients had good or excellent outcome after ultrasound-guided tendon fenestration (-Fig. 1) ${ }^{26}$ It has also been shown that the use of corticosteroid injection does not improve the results of fenestration. Its routine use is questioned given that tendon fenestration promotes healing through inflammation, which may theoretically be inhibited when combined with the injection of anti-inflammatory drugs. ${ }^{25}$ In addition, there is a theoretical increase risk of tendon rupture with the addition of corticosteroids. $^{16}$

Other tendons that have been treated with ultrasoundguided fenestration include the patellar ( $\mathbf{- F i g}$. 4), Achilles, and various tendons about the pelvis and hip (-Figs. 2 and 3) ${ }^{24}$ There are few results published in the peer-reviewed literature, but ultrasound-guided fenestration of the patellar tendon has shown to be effective with $72 \%$ of patients reporting good or excellent improvement in symptoms. ${ }^{27}$ Similarly, ultrasound-guided tendon fenestration has been effective for other tendons as well. ${ }^{24}$

\section{Technical Aspects}

\section{Preprocedural Instructions}

Prior to the fenestration procedure, the patient is instructed to avoid nonsteroidal anti-inflammatory medication for 2 weeks before and after the procedure. Avoidance of such medications theoretically may increase the chance of healing in that inflammation, growth factors, and the healing cascade will not be altered.

\section{Procedure}

Ultrasound is first performed to confirm the presence of tendinosis and to plan the optimal approach to needle guidance. The area of tendinosis is targeted, which is usually near its enthesis or attachment to bone. The needle typically is inserted along the long axis of the tendon, parallel to the transducer. Passing the needle in plane, or long axis to the transducer, is the most accurate method for ultrasound guidance because the needle is visualized in its entirety. The needle can then be angle corrected during the procedure to provide real-time visualization of the fenestration. ${ }^{28}$ The 
skin is then marked to indicate the proposed needle puncture site as well as the position of the transducer. This indicates the area that needs to be cleansed prior to the procedure, and additionally, it allows the transducer to be returned to the exact imaging plane.

First, the skin is scrubbed with an appropriate cleansing agent and sterile drapes are placed around the puncture site. The ultrasound probe is then inserted into a sterile probe cover with gel, and sterile gel is used on the skin surface. Using a 25-gauge needle, the subcutaneous tissues are infiltrated with local anesthetic. Regarding the size of the needle used for fenestration, we use a 20-gauge needle for the shoulder, hip, and knee, and a 22-gauge needle for tendons about smaller joints. Needle gauges ranging from 18 to 22 have been described in the literature. The length of the needle may be either $1.5 \mathrm{~cm}$ or $3 \mathrm{~cm}$.

A few technical aspects should be considered prior to entering the tendon to minimize complications and patient discomfort. First, the needle should be positioned in plane, along the long axis of the transducer and tendon (-Fig. 1a). Second, the needle should be placed at the proper angle while the needle is still at a very superficial level within the subcutaneous tissues. It is much easier to make changes in the needle trajectory while the needle is superficial. After entering deeper structures, such as muscle or tendon, the needle becomes more difficult to redirect. Once the needle is identified in plane and the target visualized, the needle is advanced to the tendon abnormality. Some patients have significant pain when the needle is at the surface of the tendon. Injection of an anesthetic agent at the tendon surface is often effective in significantly reducing symptoms. The amount of anesthetic agent should be minimized because it may potentially interfere with the healing process after fenestration. If the needle has a stylet, it should be removed prior to fenestration.

The needle is then passed into the tendon within the area of tendinosis, subsequently withdrawn out of the tendon, and sequentially redirected shallower as well as deeper to cover the area of tendinosis within the plane of the transducer. If the tendon abnormality is adjacent to bone, the needle is advanced to make contact with the bone. The transducer is turned 90 degrees to determine if the needle needs to be positioned more medially or laterally relative to the short axis of the tendon. To redirect the needle, the needle is retracted out of the tendon and then moved either medially or laterally. The transducer is then turned 90 degrees again in plane with the needle, and the fenestration continues in a similar manner. If the patient is having symptoms, additional anesthetic agent may be injected into the tendon, although this should be minimized. The number of times the needle passes through the tendon varies but often ranges from 15 to 30 passes, depending on the size of the tendon abnormality. As the needle passes through the abnormal tendon, the tendon tends to soften. The procedure is terminated when the entire area of tendinosis is treated and feels soft during needle advancement.

\section{Postprocedure Considerations}

After the tendon fenestration, the patient is asked to avoid nonsteroidal anti-inflammatory drugs for 2 weeks so as not to interfere with the healing process. For similar reasons, ice is avoided so as to not inhibit the induced inflammation, which is a precursor for tendon healing. For weightbearing tendons, precautions should be considered to enhance healing and avoid the complication of tendon tear. A patellar tendon tear after ultrasound-guided tendon fenestration has been described in the literature. Such negative outcomes may be due to inadequate postprocedure care or patient noncompliance. ${ }^{27}$

Consultation with the orthopedic surgeon, physiatrist, or sports medicine physician is recommended to determine the proper precautions and follow-up after tendon fenestration. For the Achilles tendon, a walking boot is often used and, for the patellar tendon, a knee brace is used. Such mechanical braces are not typically used for the upper extremity or greater trochanteric region. The timing of stretching and physical therapy after tendon fenestration is also variable in the literature, although many authors advocate waiting 2 weeks after fenestration.

\section{Contraindications}

There are several contraindications to consider when performing any ultrasound-guided percutaneous procedure. These include (1) patients with a bleeding disorder, (2) patients who are anticoagulated, and (3) the presence of local infection. The presence of underlying tendon tear deserves discussion because the risk of tendon rupture as a complication of the procedure increases with the degree of a preexisting tendon tear. One must consider the potential risk versus benefit. Some argue that a high-grade tear may not benefit from tendon fenestration but rather may be best treated with injection of autologous whole blood or PRP, although this is inconclusive in the literature. We consider fenestration with tendinosis, interstitial tearing, or partialthickness tearing up to $50 \%$ of the tendon thickness, and we avoid fenestration if a tear is $>50 \%$ of the tendon thickness.

\section{Future Directions}

The literature has shown the potential positive effects of ultrasound-guided tendon fenestration. What is unknown is which factors influence the outcome of the procedure. For example, does one specific tendon have a better outcome compared with another? Does increased vascularity, echogenicity, or size of the tendon abnormality at ultrasound influence results? With respect to the patellar tendon, it has been shown that the presence or absence of hyperemia on color Doppler imaging had no effect on patient short-term outcome (unpublished data). It is also unknown whether chronicity of the symptoms, prior treatments, and patient variables such as age or smoking affects outcome. With regard to technique, it is unknown whether needle choice or number of needle passes through the tendon has an effect. Lastly, because other percutaneous ultrasound-guided tendon injections such as hyperosmolar dextrose, autologous whole blood, and PRP also involve tendon fenestration during the procedure, does tendon fenestration alone produce similar results compared with these tendon injections? 


\section{Conclusion}

Ultrasound-guided tendon fenestration has been shown to be effective for the treatment of tendinosis at several locations within the body. Further research is needed to determine which patients would best benefit from this procedure and how tendon fenestration alone compares with other ultrasound-guided tendon injections. Regardless, ultrasoundguided tendon fenestration can be considered a potential treatment for tendinosis.

\section{References}

1 Buck FM, Grehn H, Hilbe M, Pfirrmann CW, Manzanell S, Hodler J. Magnetic resonance histologic correlation in rotator cuff tendons. J Magn Reson Imaging 2010;32(1):165-172

2 Kjellin I, Ho CP, Cervilla V, et al. Alterations in the supraspinatus tendon at MR imaging: correlation with histopathologic findings in cadavers. Radiology 1991;181(3):837-841

3 Potter HG, Hannafin JA, Morwessel RM, DiCarlo EF, O'Brien SJ, Altchek DW. Lateral epicondylitis: correlation of MR imaging, surgical, and histopathologic findings. Radiology 1995;196(1): 43-46

4 Khan KM, Bonar F, Desmond PM, et al; Victorian Institute of Sport Tendon Study Group. Patellar tendinosis (jumper's knee): findings at histopathologic examination, US, and MR imaging. Radiology 1996;200(3):821-827

5 Fearon AM, Scarvell JM, Cook JL, Smith PN. Does ultrasound correlate with surgical or histologic findings in greater trochanteric pain syndrome? A pilot study. Clin Orthop Relat Res 2010; 468(7):1838-1844

6 Aström M, Gentz CF, Nilsson P, Rausing A, Sjöberg S, Westlin N. Imaging in chronic Achilles tendinopathy: a comparison of ultrasonography, magnetic resonance imaging and surgical findings in 27 histologically verified cases. Skeletal Radiol 1996;25(7): 615-620

7 Lee KS, Wilson JJ, Rabago DP, Baer GS, Jacobson JA, Borrero CG. Musculoskeletal applications of platelet-rich plasma: fad or future? AJR Am J Roentgenol 2011;196(3):628-636

8 Jacobson JA, Lancaster S, Prasad A, van Holsbeeck MT, Craig JG, Kolowich P. Full-thickness and partial-thickness supraspinatus tendon tears: value of US signs in diagnosis. Radiology 2004; 230(1):234-242

9 Opsha O, Malik A, Baltazar R, et al. MRI of the rotator cuff and internal derangement. Eur J Radiol 2008;68(1):36-56

10 Ohberg L, Lorentzon R, Alfredson H. Neovascularisation in Achilles tendons with painful tendinosis but not in normal tendons: an ultrasonographic investigation. Knee Surg Sports Traumatol Arthrosc 2001;9(4):233-238

11 Zanetti M, Metzdorf A, Kundert HP, et al. Achilles tendons: clinical relevance of neovascularization diagnosed with power Doppler US. Radiology 2003;227(2):556-560

12 de Vos RJ, Weir A, Cobben LP, Tol JL. The value of power Doppler ultrasonography in Achilles tendinopathy: a prospective study. Am J Sports Med 2007;35(10):1696-1701
13 Coombes BK, Bisset L, Vicenzino B. Efficacy and safety of corticosteroid injections and other injections for management of tendinopathy: a systematic review of randomised controlled trials. Lancet 2010;376(9754):1751-1767

14 Zissen MH, Wallace G, Stevens KJ, Fredericson M, Beaulieu CF. High hamstring tendinopathy: MRI and ultrasound imaging and therapeutic efficacy of percutaneous corticosteroid injection. AJR Am J Roentgenol 2010;195(4):993-998

15 Labrosse JM, Cardinal E, Leduc BE, et al. Effectiveness of ultrasound-guided corticosteroid injection for the treatment of gluteus medius tendinopathy. AJR Am J Roentgenol 2010;194(1):202-206

16 MacMahon PJ, Eustace SJ, Kavanagh EC. Injectable corticosteroid and local anesthetic preparations: a review for radiologists. Radiology 2009;252(3):647-661

17 Distel LM, Best TM. Prolotherapy: a clinical review of its role in treating chronic musculoskeletal pain. PM R 2011;3(6, Suppl 1): S78-S81

18 Maxwell NJ, Ryan MB, Taunton JE, Gillies JH, Wong AD. Sonographically guided intratendinous injection of hyperosmolar dextrose to treat chronic tendinosis of the Achilles tendon: a pilot study. AJR Am J Roentgenol 2007;189(4):W215-W220

19 Kampa RJ, Connell DA. Treatment of tendinopathy: is there a role for autologous whole blood and platelet rich plasma injection? Int J Clin Pract 2010;64(13):1813-1823

20 Sheth U, Simunovic N, Klein G, et al. Efficacy of autologous plateletrich plasma use for orthopaedic indications: a meta-analysis. J Bone Joint Surg Am 2012;94(4):298-307

21 Thanasas C, Papadimitriou G, Charalambidis C, Paraskevopoulos I, Papanikolaou A. Platelet-rich plasma versus autologous whole blood for the treatment of chronic lateral elbow epicondylitis: a randomized controlled clinical trial. Am J Sports Med 2011; 39(10):2130-2134

22 Finnoff JT, Fowler SP, Lai JK, et al. Treatment of chronic tendinopathy with ultrasound-guided needle tenotomy and platelet-rich plasma injection. PM R 2011;3(10):900-911

23 James SL, Ali K, Pocock C, et al. Ultrasound guided dry needling and autologous blood injection for patellar tendinosis. Br J Sports Med 2007;41(8):518-521; discussion 522

24 Housner JA, Jacobson JA, Misko R. Sonographically guided percutaneous needle tenotomy for the treatment of chronic tendinosis. J Ultrasound Med 2009;28(9):1187-1192

25 McShane JM, Shah VN, Nazarian LN. Sonographically guided percutaneous needle tenotomy for treatment of common extensor tendinosis in the elbow: is a corticosteroid necessary? J Ultrasound Med 2008;27(8):1137-1144

26 McShane JM, Nazarian LN, Harwood MI. Sonographically guided percutaneous needle tenotomy for treatment of common extensor tendinosis in the elbow. J Ultrasound Med 2006;25(10): 1281-1289

27 Housner JA, Jacobson JA, Morag Y, Pujalte GG, Northway RM, Boon TA. Should ultrasound-guided needle fenestration be considered as a treatment option for recalcitrant patellar tendinopathy? A retrospective study of 47 cases. Clin J Sport Med 2010;20(6): 488-490

28 Joines MM, Motamedi K, Seeger LL, DiFiori JP. Musculoskeletal interventional ultrasound. Semin Musculoskelet Radiol 2007; 11(2):192-198 